\title{
Asset allocation with conditional value-at-risk budgets
}

\section{Kris Boudt}

Faculty of Business, Vrije Universiteit Brussel, Pleinlaan 2, 1050 Brussels, Belgium; email: kris.boudt@vub.ac.be

and

Faculty of Economics and Business, VU University Amsterdam, de Boelelaan 1105, 1081 HV Amsterdam, The Netherlands

\section{Peter Carl}

William Blair \& Company, 222 West Adams Street, Chicago, IL 60606, USA;

email: pcarl@gsb.uchicago.edu

\section{Brian G. Peterson}

DV Trading, 216 W Jackson Boulevard, Chicago, IL 60606, USA;

email: brian@braverock.com

(Received October 19, 2011; revised May 24, 2012; accepted June 11, 2012)

\begin{abstract}
Risk budgets are frequently used to allocate the risk of a portfolio by decomposing the total portfolio risk into the risk contribution of each component position. Many approaches to portfolio allocation use ex post methods for constructing risk budgets and take the variance as a risk measure. In this paper, however, we use ex ante methods to evaluate the component contribution to conditional value-at-risk (CVaR) and allocate risk. The proposed minimum CVaR concentration portfolio draws a balance between the investor's return objectives and the diversification of risk across the portfolio. For a portfolio invested in bonds, commodities, equities and real estate, we find that over the period from January 1984 to June 2010, the minimum CVaR concentration portfolio offers an attractive compromise between the good risk-adjusted return properties of the minimum CVaR portfolio and the positive return potential and low portfolio turnover of an equally weighted portfolio.
\end{abstract}

\footnotetext{
The authors thank David Ardia, Bernhard Pfaff, Dale Rosenthal and two reviewers for helpful comments. Financial support from the National Bank of Belgium is gratefully acknowledged. The code to replicate the analysis is available in the $\mathrm{R}$ packages PerformanceAnalytics (Carl and Peterson 2010) and PortfolioAnalytics (Boudt et al 2011). More information on the utilization of these packages for the estimation and optimization of portfolio risk budgets can be found at www.econ.kuleuven.be/ kris.boudt/public/riskbudgets.htm.
} 


\section{INTRODUCTION}

Risk budgets are frequently used to allocate the risk of a portfolio by decomposing the total portfolio risk into the risk contribution of each component position. While volatility-weighted portfolio allocation methods have existed for many years, the ex ante use of risk budgets in portfolio allocation is more recent.

The risk parity portfolio of Qian (2005) allocates the portfolio variance equally across the portfolio components. Maillard et al (2010) show that the volatility of this portfolio is located between the volatilities of the minimum variance and the equally weighted portfolio. Zhu et al (2010) study optimal mean-variance portfolio selection under a direct constraint on the contributions to portfolio variance. Compared with the equally weighted portfolio, the most diversified portfolio of Choueifaty and Coignard (2008) and the global minimum variance portfolio, Lee (2011) finds that the riskcontribution approach is

potentially more applicable because of its heuristic nature, economic intuition, and the financial interpretation that ties its concept to economic losses.

However, to our knowledge, the literature on risk contribution portfolios lacks a detailed study on the use of downside risk budgets rather than portfolio variance budgets as an ex ante portfolio allocation tool. Given the nonnormality of many financial return series, the practice of assigning equal weights to positive and negative returns when computing risk contributions is likely to be suboptimal with respect to the allocation of risk through ex ante downside risk budgets. We fill this gap by explaining in detail the implementation of portfolio strategies that use downside risk contributions rather than variance contributions as an objective or constraint.

Our focus is on portfolio conditional value-at-risk (CVaR), since unlike value-atrisk (VaR), CVaR has all the properties a risk measure requires to be coherent and is a convex function of the portfolio weights (Artzner et al 1999; Pflug 2000). Moreover, CVaR provides less incentive to load on to tail risk above the VaR level. By integrating the CVaR budget into their optimal portfolio policy, investors can directly optimize downside risk diversification.

For many practical applications, the risk parity constraint that requires all assets to contribute equally to portfolio risk is too restrictive. As an alternative, we propose to minimize the largest $\mathrm{CVaR}$ risk contribution in the portfolio. Unconstrained, this portfolio criterion still generates portfolios that are similar to the risk parity portfolio, but it has the advantage that it can be more easily combined with many other investor objectives and constraints (such as return targets or drawdown and cardinality constraints).

The outline of the paper is as follows. First, we review the definition and estimation of CVaR portfolio budgets in Section 2. In Section 3 we describe several portfolio 
allocation strategies that use the portfolio component $C$ VaR risk budget as an objective or constraint in the portfolio optimization problem. We conclude with a thorough performance study of the use of CVaR allocation methodology to find the optimal mix of bonds, commodities, equities and real estate. Portfolios are rebalanced quarterly, taking into account the salient features of financial return data, such as time-varying volatility and correlation, skewness and heavy tails. We find that over the period 1984-2010, the minimum CVaR concentration (MCC) portfolio offers an attractive compromise between the good risk-adjusted return properties of the minimum CVaR portfolio and the positive return potential and low portfolio turnover of an equally weighted portfolio.

\section{PORTFOLIO CONDITIONAL VALUE-AT-RISK BUDGETS}

\subsection{Definition}

The first step in the construction of a risk budget is to define how portfolio risk and its risk contributions should be measured. A naive approach is to set the risk contribution equal to the stand-alone risk of each portfolio component. This approach is overly simplistic and neglects important diversification or multiplication effects of the component units being exposed differently to the underlying risk factors. Using game theory, Denault (2001) has shown that the only satisfactory risk allocation principle is to measure the risk contribution as the weight of the position in the portfolio times the partial derivative of the portfolio risk $R_{w}$ with respect to that weight:

$$
C_{(i)} R_{w}=w_{(i)} \frac{\partial R_{w}}{\partial w_{(i)}} .
$$

The standard deviation, VaR and CVaR of a portfolio are all linear in position size. By Euler's theorem, we have that for such risk measures the total portfolio risk equals the sum of the risk contributions in (2.1).

Chow and Kritzman (2001), Litterman (1996), Maillard et al (2010), Peterson and Boudt (2008) and Scherer (2007) study the use of portfolio standard deviation and VaR budgets. In his book Risk Budgeting, Pearson (2002, p. 7) notes that

value-at-risk has some well known limitations, and it may be that some other risk measure eventually supplants value-at-risk in the risk budgeting process.

We develop a risk budgeting framework for portfolio CVaR. Portfolio CVaR can be expressed in monetary value or percentage returns. Our goal is to apply the CVaR budget in an investment strategy based on quantitative analysis of the asset returns. We therefore choose to define CVaR in percentage returns.

Denote by $r_{w t}$ the return at time $t$ on the portfolio with weight vector $w$. To simplify notation, we omit the time index $t$ whenever no confusion is possible and assume that 
the density function of $r_{w}$ is continuous. At a preset probability level denoted by $\alpha$, which is typically set between $1 \%$ and $5 \%$, the portfolio VaR is the negative value of the $\alpha$ quantile of the portfolio returns. The portfolio CVaR is the negative value of the expected portfolio return when that return is less than its $\alpha$ quantile:

$$
\operatorname{CVaR}_{w}(\alpha)=-\mathbb{E}\left[r_{w} \mid r_{w} \leqslant-\operatorname{VaR}_{w}(\alpha)\right]
$$

with $\mathrm{E}$ the expectation operator. The $\mathrm{CVaR}$ contribution is the weight of the position in the portfolio times the partial derivative of the portfolio CVaR with respect to that weight:

$$
C_{(i)} \operatorname{CVaR}_{w}(\alpha)=w_{(i)} \frac{\partial \operatorname{CVaR}_{w}(\alpha)}{\partial w_{(i)}},
$$

where $w_{(i)}$ is the portfolio weight of position $i$ and there are $N$ assets in the investment universe $(i=1, \ldots, N)$. For ease of interpretation, the CVaR contributions are standardized by the total CVaR. This yields the percentage CVaR contributions:

$$
\% C_{(i)} \operatorname{CVaR}_{w}(\alpha)=\frac{C_{(i)} \operatorname{CVaR}_{w}(\alpha)}{\operatorname{CVaR}_{w}(\alpha)} .
$$

The CVaR contributions are directly linked to the downside risk concentration of the portfolio. Scaillet (2002) shows that the contributions to CVaR correspond to the conditional expectation of the return of the portfolio component when the portfolio loss is larger than its VaR loss:

$$
C_{(i)} \operatorname{CVaR}_{w}(\alpha)=-\mathrm{E}\left[w_{(i)} r_{(i)} \mid r_{w} \leqslant-\operatorname{VaR}_{w}(\alpha)\right]
$$

An interesting summary statistic of the portfolio's CVaR allocation is what we call the portfolio CVaR concentration, defined as the largest component $\mathrm{CVaR}$ of all positions:

$$
C_{w}(\alpha)=\max _{i} C_{(i)} \operatorname{CVaR}_{w}(\alpha)
$$

As we will show later, minimizing the portfolio CVaR concentration leads to portfolios with a relatively low CVaR and a balanced CVaR allocation.

\subsection{Estimation}

The actual risk contributions can be estimated in two ways. One approach is to estimate the risk contributions by replacing the expectation in (2.5) with the sample counterpart evaluated using historical or simulated data. In a portfolio optimization setting, the risk contributions need to be evaluated for a large number of possible weights, and therefore fast and explicit estimators are needed. A more elegant approach for 
optimization problems is therefore to derive the analytical formulas of the risk contributions. If the returns at time $t$ are conditionally normally distributed with mean $\mu_{t}$ and covariance matrix $\Sigma_{t}$, then $\mathrm{CVaR}$ at time $t$ is given by

$$
\operatorname{CVaR}_{w}(\alpha)=-w^{\prime} \mu_{t}+\sqrt{w^{\prime} \Sigma_{t} w} \frac{\phi\left(z_{\alpha}\right)}{\alpha},
$$

with $z_{\alpha}$ the $\alpha$ quantile of the standard normal distribution and $\phi$ the standard normal density function. The contribution to CVaR is then

$$
C_{(i)} \operatorname{CVaR}_{w}(\alpha)=w_{(i)}\left[-\mu_{(i) t}+\frac{\left(\Sigma_{t} w\right)_{(i)}}{\sqrt{w^{\prime} \Sigma_{t} w}} \frac{\phi\left(z_{\alpha}\right)}{\alpha}\right] .
$$

Financial returns are usually nonnormally distributed. In the empirical application, we use the modified CVaR (contribution) estimator proposed by Boudt et al (2008). Based on Cornish-Fisher expansions, the modified CVaR estimate is an explicit function of the components of the underlying asset returns. It has been shown to deliver accurate estimates of $\mathrm{CVaR}$ (contributions) for portfolios with nonnormal returns. For the exact definition of this estimator, refer to Appendix A. The Cornish-Fisher-based estimates of CVaR work well for tail probabilities that are not too small, as shown by Boudt et al (2008). Throughout the paper, we set the loss probability $\alpha$ to 5\%. This is a common choice in practical portfolio optimizations, as mentioned, for example, by Keel and Ardia (2011) and Topaloglou et al (2002). For smaller values of $\alpha$, the Cornish-Fisher CVaR estimator becomes less reliable and eventually breaks down. A detailed study of the sensitivity of the portfolio performance to the choice of $\alpha$ is left for further research.

\section{CONDITIONAL VALUE-AT-RISK BUDGETS IN PORTFOLIO OPTIMIZATION}

Previously, risk budgets based on portfolio standard deviation and VaR have been used as either an ex post or an ex ante tool for tuning the portfolio allocation.

In the ex post approach, the portfolio is first optimized without taking into account the risk allocation. The risk budget of the optimal portfolio is then estimated and risk budget violations are adjusted on a marginal basis. The rationale for this is that the risk contributions in (2.1) can be interpreted as the marginal risk impact of the corresponding position. Because of transaction costs, traders and portfolio managers often update their portfolios incrementally, which makes the marginal interpretation of risk contribution useful in practice (Litterman 1996; Stoyanov et al 2009). When the risk contribution of a position is zero, Litterman (1996) calls this the "best hedge" position for that portfolio component. The positions with the largest risk contributions are called "hot spots". If a risk contribution is negative, a small increase in the 
corresponding portfolio weight leads to a decrease in the portfolio risk. Keel and Ardia (2011), however, show that reallocation of the portfolio based on these risk contributions is limited in two ways. First of all, as a sensitivity measure, the risk contributions are precise for infinitesimal changes, but these approximations can be poor for realistic reallocations. Second, they assume changes in a single position while keeping all other positions fixed. In the presence of a full investment constraint, this is unrealistic.

While various volatility-weighted portfolio allocation methods have existed for many years, the ex ante use of risk budgets in portfolio allocation is more recent. Qian's (2005) "risk parity portfolio" allocates portfolio variance equally across the portfolio components. Maillard et al (2010) call this the "equally-weighted risk contribution portfolio" or, simply, the equal-risk contribution (ERC) portfolio. They derive the theoretical properties of the ERC portfolio and show that its volatility is located between those of the minimum variance and equal-weight portfolios. Zhu et al (2010) study optimal mean-variance portfolio selection under a direct constraint on the contributions to portfolio variance.

Our first contribution to the recent literature is to use (percentage) CVaR contributions rather than variance contributions as an objective or constraint in portfolio optimization. By integrating the CVaR budget into their optimal portfolio policy, investors can directly optimize downside risk diversification. The rationale for this is the result in (2.5) that the CVaR contributions correspond to the conditional expectation of the return of the portfolio component when the portfolio loss is larger than its VaR loss. From (2.3) and (2.5), it also follows that the percentage CVaR contribution can be rewritten as the ratio of the expected return on the position at the time the portfolio experiences a beyond-VaR loss to the expected value of the beyond-VaR portfolio losses:

$$
\% C_{(i)} \operatorname{CVaR}_{w}(\alpha)=\frac{\mathrm{E}\left[w_{(i)} r_{(i)} \mid r_{w} \leqslant-\operatorname{VaR}_{w}(\alpha)\right]}{\mathrm{E}\left[r_{w} \mid r_{w} \leqslant-\operatorname{VaR}_{w}(\alpha)\right]} .
$$

In almost all practical cases, the denominator in (3.1) is negative, so that a high positive percentage CVaR contribution indicates that the position has a large loss when the portfolio also has a large loss. The higher the percentage CVaR, the more the portfolio downside risk is concentrated on that asset and vice versa.

Our second contribution is the proposal of two strategies for the use of CVaR budgets in portfolio optimization in order to balance the maximum return, minimum downside risk and maximum downside risk diversification objectives of an investor. The first strategy is the MCC portfolio, which uses the downside risk diversification criterion as an objective rather than a constraint. More formally, the MCC portfolio allocation is given by

$$
w^{\mathrm{MCC}}=\underset{w \in W}{\operatorname{argmin}} C_{w}(\alpha),
$$


with the portfolio's CVaR concentration $C_{w}(\alpha)$ as defined in (2.6). $W$ is the set of feasible portfolio weights. Unless otherwise mentioned, $W$ is only restricted by a full investment constraint.

The second strategy consists of imposing bound constraints on the percentage CVaR contributions. This may be viewed as a direct substitute for a risk diversification approach based on position limits. It has the ERC constraint as a special case:

$$
\% C_{(1)} \operatorname{CVaR}_{w}(\alpha)=\cdots=\% C_{(N)} \operatorname{CVaR}_{w}(\alpha)=1 / N .
$$

Note that that for a portfolio that has the ERC property, the relative weights are inversely proportional to the marginal impact of the position on the portfolio CVaR:

$$
\frac{w_{(i)}}{w_{(j)}}=\frac{\partial \operatorname{CVaR}_{w}(\alpha) / \partial w_{(j)}}{\partial \operatorname{CVaR}_{w}(\alpha) / \partial w_{(i)}} .
$$

It follows that the ERC allocation strategy yields portfolios that give higher weights to assets with a small marginal risk impact and downweights the investments with a high marginal risk (the so-called hot spots in Litterman (1996)).

In the following subsections, we study the properties of these two approaches in more detail. In the empirical section, we will compare these CVaR budget-based portfolio allocation rules with the more standard minimum CVaR (MC) and equalweight $(\mathrm{EW})$ portfolios:

$$
w^{\operatorname{minCVaR}}=\operatorname{argmin}_{w \in W} \operatorname{CVaR}_{w}(\alpha) \quad \text { and } \quad w^{\mathrm{EW}}=(1 / N, \ldots, 1 / N)^{\prime} .
$$

\subsection{Properties of the MCC portfolio}

For the derivation of the properties of the MCC portfolio, it is useful to rewrite the portfolio CVaR concentration as the portfolio CVaR multiplied by the largest percentage $\mathrm{CVaR}$ contribution:

$$
C_{w}(\alpha)=\mathrm{CVaR}_{w}(\alpha) \max \left\{\% C_{(1)} \operatorname{CVaR}_{w}(\alpha), \ldots, \% C_{(N)} \operatorname{CVaR}_{w}(\alpha)\right\} .
$$

The first factor in (3.6) is minimized by the minimum CVaR portfolio. The second factor attains its lowest value when the portfolio has the ERC property, since

$$
\max \left\{\% C_{(1)} \mathrm{CVaR}_{w}(\alpha), \ldots, \% C_{(N)} \mathrm{CVaR}_{w}(\alpha)\right\} \geqslant 1 / N \text {. }
$$

By minimizing the product of these two factors, the MCC portfolio strikes a balance between the objectives of portfolio risk diversification and total risk minimization. Compared with the unconstrained minimum $\mathrm{CVaR}$ portfolio, we obtain that the $\mathrm{CVaR}$ of the MCC portfolio is higher, but the risk is less concentrated. In fact, we show in 
Appendix A that the percentage CVaR of the fully invested minimum CVaR portfolio coincides with the component's portfolio weight:

$$
\% C_{(i)} \operatorname{CVaR}_{w^{\min C V a R}}(\alpha)=w_{(i)}^{\min C V a R} .
$$

It is well-known that the minimum CVaR portfolio generally suffers from the drawback of portfolio concentration. By (3.7), this carries directly over to the CVaR allocation.

In many cases, the $\mathrm{CVaR}$ concentration is not a convex function of the portfolio weights, and $C_{w}(\alpha)$ may also not be differentiable. For this reason, we recommend the use of a derivative-free global optimizer to find the MCC portfolio. We used the differential evolution algorithm developed by Price et al (2005). Ardia et al (2011) provide an example of how to implement the MCC portfolio.

The MCC objective can easily be combined with a return target. This serves the general purpose of maximizing return subject to some level of risk, while also minimizing the risk concentration at that risk level. We define the mean-CVaR concentration efficient frontier as the collection of all portfolios that achieve the lowest degree of $\mathrm{CVaR}$ concentration for a return objective. For a given return target $\bar{r}$, the mean-CVaR concentration efficient portfolio solves

$$
\min _{w \in W} C_{w}(\alpha) \quad \text { such that } w^{\prime} \mu \geqslant \bar{r} .
$$

The minimum CVaR portfolio under the ERC constraint in (3.4) is an alternative to the MCC portfolio for attaining a balance between the objectives of portfolio risk diversification and total risk minimization. In our data examples, the two portfolios were always very similar.

The advantage of the MCC portfolio over the ERC constrained minimum CVaR portfolio is that it is computationally simpler and also will yield a solution if the ERC constraint is not feasible or conflicts with other constraints. Since most real-world portfolios are constructed with an explicit or implicit return objective and other constraints, the ability to combine with other objectives and constraints is an important consideration for asset managers that is often incompatible with the published literature on utilizing risk metrics in portfolio construction. Note also that the properties of the MCC portfolio generalize to any minimum risk concentration portfolio, as long as the portfolio risk measure is a homogeneous function of order one of the portfolio weights.

\subsection{Portfolio allocation under CVaR allocation constraints}

The risk allocation can also be controlled by imposing explicit constraints on the percentage CVaR allocations. This process operates in much the same way that portfolio managers impose weight constraints on portfolios. 
FIGURE 1 Percentage CVaR contribution of asset 1 as a function of its portfolio weight for a two-asset portfolio with asset returns that have a bivariate normal distribution with means $\mu_{1}$ and $\mu_{2}$, correlation $\rho$ and standard deviations $\sigma_{1}$ and $\sigma_{2}$, respectively.

(a)

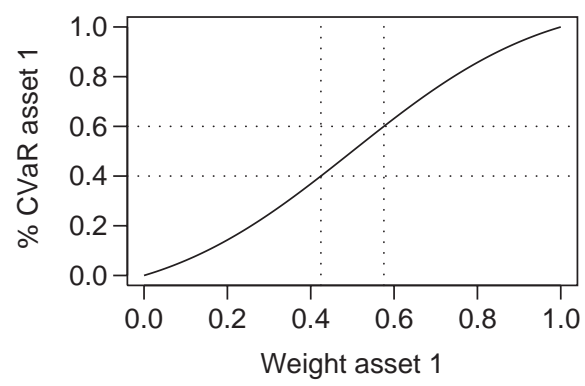

(c)

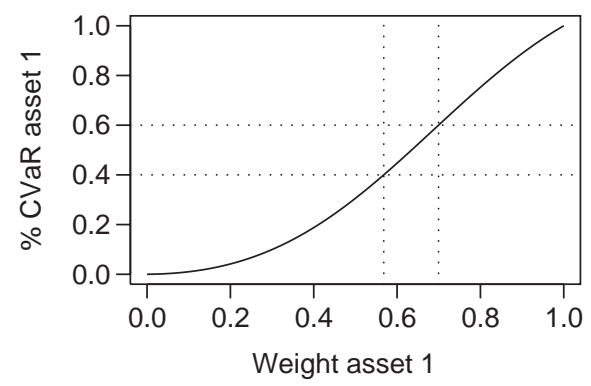

(b)

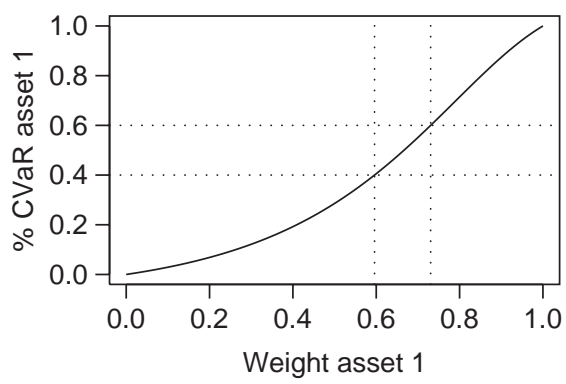

(d)

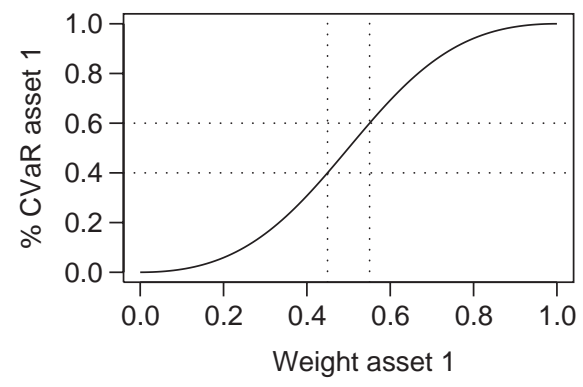

(a) $\mu_{1}=\mu_{2}=0, \sigma_{1}=\sigma_{2}=1$ and $\rho=0.5$. (b) $\mu_{1}=\mu_{2}=0, \sigma_{1}=1, \sigma_{2}=2$ and $\rho=0.5$. (c) $\mu_{1}=1, \mu_{2}=0$, $\sigma_{1}=\sigma_{2}=1$ and $\rho=0.5$. (d) $\mu_{1}=\mu_{2}=0, \sigma_{1}=\sigma_{2}=1$ and $\rho=0$.

Such percentage CVaR contribution constraints reduce the feasible space in a way that depends on the return characteristics. Stoyanov et al (2009) study in detail the effect of the component return characteristics on the total portfolio CVaR. To build further intuition via a stylized example, we plot in Figure 1 the percentage CVaR contributions for a two-asset portfolio with asset returns that have a bivariate normal distribution with means $\mu_{1}$ and $\mu_{2}$, standard deviations $\sigma_{1}$ and $\sigma_{2}$ and a correlation $\rho$. Of course, the percentage $\mathrm{CVaR}$ contribution is 0 and 1 , respectively, if the weight is 0 and 1 . In between these values, the percentage CVaR displays an S-shape.

The dotted lines in Figure 1 illustrate the effect on the feasible space for portfolio weight 1 of imposing an upper $60 \%$ bound on the percentage CVaR contributions of the two assets. This implies that the percentage CVaR contribution of asset 1 has to be between $40 \%$ and $60 \%$. In parts (a) and (d), the two assets are identical. In this case, 
the feasible space is centered around the equal-weight portfolio. For $\rho=0$ (part (d)), the percentage CVaR contribution of asset 1 as a function of its weight is more curved than that for $\rho=0.5$ (part (a)).

In the other figures, asset 1 is more attractive than asset 2 since it has either a lower volatility or a higher expected return. We see that this leads to a shift of the feasible space to the right, with allows portfolio weights around $60 \%$. The set of possible weights satisfying the box constraints on the percentage CVaR contributions changes in an intuitively appealing way when differences in return and volatility are allowed.

For general portfolios with nonnormal returns, there is no explicit representation of the percentage CVaR constraint as a weight constraint available for investment. A general purpose portfolio solver that can handle such percentage CVaR contribution constraints is available in the R package PortfolioAnalytics of Boudt et al (2011).

\section{EMPIRICAL RESULTS}

In this section, we apply the CVaR decomposition methodology to optimize portfolios that allocate across asset classes. The analysis is based on the January 1976-June 2010 monthly total USD returns of broad bond, commodity, equity and real estate asset class indexes, namely the Merrill Lynch Domestic Master index, the S\&P Goldman Sachs commodity index, the S\&P 500 index and the National Association of Real Estate Investment Trusts (NAREIT) index. The data is obtained from Datastream. We will start with a static two asset bond-equity portfolio, and expand to a larger portfolio to study the effects of rebalancing under various constraints and objectives. We impose in all portfolio allocations the full investment constraint and exclude short sales.

Because of the nonnormality in the data, we use the modified CVaR estimator of Boudt et al (2008). Its implementation requires an estimate of the first four moments of the portfolio returns. For the in-sample analysis in Section 2, all moments are estimated by their historical sample counterpart on the Winsorized data using the method of Boudt et al (2008). Over the January 1976-June 2010 period, the annualized average monthly returns (standard deviations) of the bond and US equity markets are $7.56 \%(5.36 \%$ ) and $10.69 \%$ (14.51\%), respectively. The 95\% CVaRs of the bond and US equity index are $2.31 \%$ and $9.34 \%$, respectively. The GSCI has a relatively low annualized monthly return $(6.22 \%)$ and high risk (annualized standard deviation of $11.84 \%$ and monthly $95 \%$ CVaR of $11.84 \%$ ). With an annualized return of $11.28 \%$, an annualized standard deviation of $14.57 \%$ and a monthly CVaR of $10.61 \%$, the NAREIT index offers a higher return than, and a similar standard deviation to, the S\&P 500 index, but its downside risk is slightly higher. 
TABLE 1 Weight and CVaR allocation of bond-equity portfolios, together with the in-sample annualized mean and monthly $95 \%$ CVaR over the period January 1976-June 2010.

\begin{tabular}{|c|c|c|c|c|c|c|c|}
\hline & \multicolumn{2}{|c|}{ Weight allocation (\%) } & \multicolumn{2}{|c|}{ CVaR allocation (\%) } & $\begin{array}{c}\text { Annual } \\
\text { mean (\%) }\end{array}$ & $\begin{array}{l}\text { Annual } \\
\text { SD (\%) }\end{array}$ & $\begin{array}{c}95 \% \\
\text { CVaR (\%) }\end{array}$ \\
\hline $60 / 40$ weight & 60 & 40 & 14.79 & 85.21 & 8.81 & 7.55 & 3.79 \\
\hline $\begin{array}{l}\text { Min CVaR } \\
\text { concentration }\end{array}$ & 77.51 & 22.49 & 50 & 50 & 8.27 & 5.68 & 2.79 \\
\hline Min CVaR & 96.18 & 3.82 & 96.18 & 3.82 & 7.68 & 5.27 & 2.28 \\
\hline
\end{tabular}




\subsection{Static bond-equity portfolio}

The simple bond-equity portfolio application in Table 1 on the preceding page illustrates the impact of the portfolio policy on the risk allocation. Portfolio managers frequently rely on the heuristic approach of applying position limits to ensure diversification. Such a simple approach may ignore the individual risks of the portfolio assets and their risk dependence. A first example is the equal-weight portfolio, which is popular in practice because it does not require any information on the risk and return and supposedly provides a diversified portfolio. A second popular positionconstrained bond-equity portfolio is the $60 / 40$ portfolio, investing $60 \%$ in bonds and $40 \%$ in equity. The first two lines in Table 1 show the estimated risk allocation of these portfolios. We see that position limits clearly fail to produce portfolios with an ex ante risk diversification: $97 \%$ and $86 \%$, respectively, of the portfolio CVaR is caused by the equity investment in the equal-weight and 60/40 portfolios.

Note also in Table 1 that the equal-weight and 60/40 portfolios have a relatively high level of total portfolio CVaR. Rockafellar and Uryasev (2000), among others, recommend the minimum CVaR portfolio to investors wanting to avoid extreme losses. For our sample, the minimum CVaR portfolio has a monthly $95 \%$ CVaR of $2.28 \%$, which is less than half the CVaR of the equal-weight portfolio. However, the portfolio risk is still heavily concentrated in one asset: the bond allocation is responsible for $96 \%$ of portfolio $\mathrm{CVaR}$ in the minimum $\mathrm{CVaR}$ portfolio.

This paper proposes the MCC portfolio for investors interested in having both a high ex ante downside risk diversification and a low total portfolio CVaR. We see in Figure 2 on the facing page that for this sample the MCC portfolio has the highest CVaR diversification possible: it is an equal risk contribution portfolio with a $22 \%$ share in equity. It has only a slightly higher $\mathrm{CVaR}$ than the minimum $\mathrm{CVaR}$ portfolio, but it also has a higher average return.

Finally, we also consider substituting the 60/40 weight allocation with a 60/40 risk allocation: $82 \%$ of this percentage risk constrained portfolio is invested in bonds. As for the MCC portfolio, the price for risk diversification is a slight increase in the portfolio CVaR compared with the minimum CVaR portfolio, but this is also compensated by a higher average return.

\subsection{Mean-CVaR concentration efficient frontier}

In comparison with the ERC portfolio of Qian (2005), the MCC portfolio has the advantage that it may be easily combined with many other investor objectives and constraints. Adding a return target to the MCC objective, we plot in Figure 2 on the facing page and Figure 3 on page 53 the mean-CVaR concentration efficient portfolios for the investment universe consisting of the US bond, S\&P 500, NAREIT and GSCI asset class indexes. These portfolios are compared with the mean-standard 
FIGURE 2 Weight and CVaR allocation of mean-standard deviation, mean-CVaR and mean-CVaR concentration efficient portfolios for various levels of annualized portfolio returns.

(a)

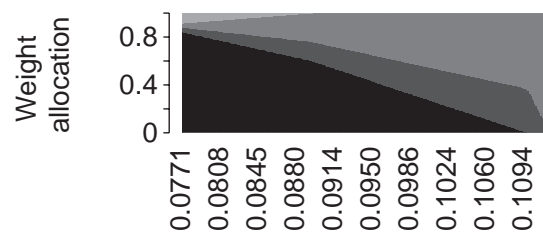

(c)

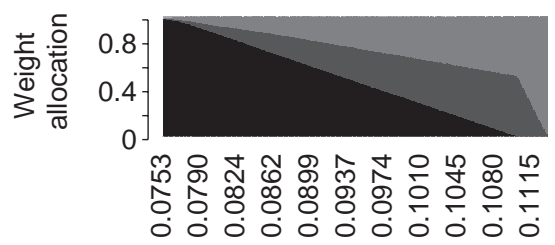

(e)

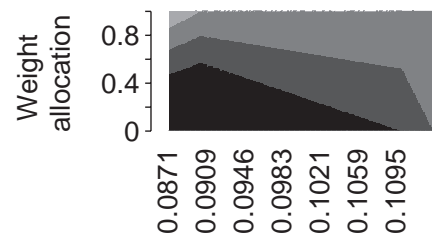

(b)

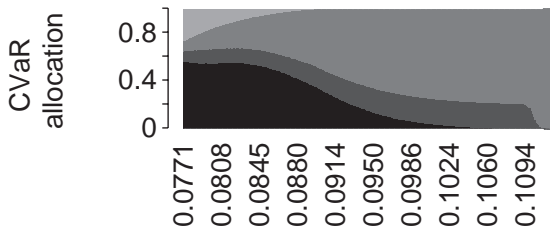

(d)

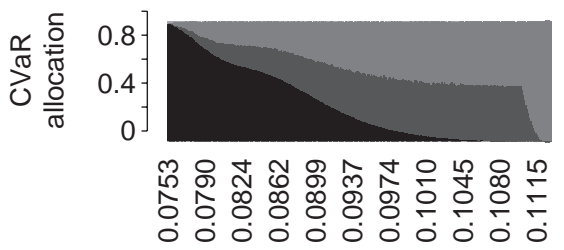

(f)

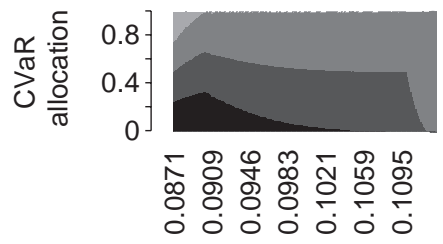

$$
\text { - US bond - S\&P } 500 \text { - NAREIT - GSCI }
$$

(a), (b) Mean standard deviation. (c), (d) Mean-CVaR. (e), (f) Mean-CVaR concentration.

deviation and mean-CVaR efficient portfolios. Part (a) of Figure 3 plots the mean-risk frontiers, while part (c) shows the annualized mean return of the portfolios against the largest percentage CVaR contribution. A joint reading of these plots is needed to understand the trade-off between the maximum return, minimum risk and minimum risk concentration objectives.

The optimal weights for the mean-CVaR concentration portfolios as a function of the return target are reported in part (e) of Figure 2. Without return constraint, the MCC portfolio invests $47.34 \%$ in the bond index, $20.70 \%$ in the S\&P 500 index, $18.44 \%$ in the NAREIT index and $13.52 \%$ in the GSCI. Part (f) of Figure 2 shows that the CVaR 
allocation of the unconstrained MCC portfolio is very close to that of an equal risk contribution portfolio. Parts (a) and (b) of Figure 2 show the weight and CVaR allocation of the classical mean-standard deviation and mean-CVaR efficient portfolios. There are several striking differences. First, the mean-standard deviation portfolio is slightly more diversified than the mean-CVaR portfolio: $97 \%$ of the unconstrained min CVaR portfolio is invested in the bond index, against $84 \%$ for the minimum standard deviation portfolio. Second, since the CVaR of the GSCI is significantly higher than the $\mathrm{CVaR}$ of the other asset class indexes, its percentage risk contribution is triple its portfolio weight. Third, the annualized returns of the minimum standard deviation and CVaR portfolios are $7.7 \%$ and $7.5 \%$, respectively, while for the MCC portfolio it is $8.7 \%$. The risk-return trade-off is visualized in Figure 3 on the facing page. We see that the equal-weight portfolio has the highest average return and risk of all unconstrained portfolios, followed by the MCC portfolio. The risk-return characteristics of the min standard deviation and min CVaR portfolios are similar.

Figure 2 on the preceding page shows that imposing a return constraint on the minimum standard deviation and CVaR portfolios leads to a higher allocation to the S\&P 500 and the NAREIT index, and a reduction in the bond and GSCI investment. Of course this leads to portfolios with a higher return and risk, but, interestingly, as long as the return target is below $9 \%$, it reduces the risk concentration of the portfolio, as can be seen from part (c) of Figure 3 on the facing page. From that point onward, the NAREIT index becomes the largest risk contributor, and higher returns are traded off with both a higher total portfolio risk and risk concentration.

The mean-CVaR concentration efficient portfolio is very different from the mean$\mathrm{CVaR}$ and mean-standard deviation efficient portfolios. On this data set, the mean$\mathrm{CVaR}$ concentration efficient frontier has three distinct segments. Unconstrained, the mean-CVaR concentration efficient frontier is an equal risk contribution portfolio with an annualized return of $8.7 \%$. For a target return between $8.7 \%$ and $9.01 \%$, the portfolio CVaR concentration increases from $0.96 \%$ to $1.19 \%$, but the portfolio CVaR decreases from $3.87 \%$ to $3.59 \%$. This is due to a reallocation of the more risky commodity investment into bonds, equity and real estate, as can be seen in Figure 2 on the preceding page. At the end of this segment, only $1 \%$ of the portfolio is invested in commodities. Bonds dominate the portfolio budget allocation, with a $58 \%$ share. On the second segment, the bond allocation shrinks to zero, while the shares of the S\&P 500 and the NAREIT index rise from $22 \%$ to $52 \%$ and from $20 \%$ to $48 \%$, respectively. On this angle portfolio, the S\&P 500 and NAREIT index each contribute $50 \%$ to the portfolio CVaR, which is now $7.0 \%$ compensated by a target return of $11 \%$. The portfolios on the final segment of the frontier gradually replace the S\&P 500 investment with the NAREIT. Since this asset offers the highest return, it is also the endpoint of the long-only constrained mean-CVaR concentration efficient frontier. 
FIGURE 3 Annualized mean return versus the annualized portfolio standard deviation, the monthly portfolio $95 \% \mathrm{CVaR}$ and the largest percentage CVaR contribution for the meanstandard deviation, mean-CVaR and mean-CVaR concentration efficient portfolios.

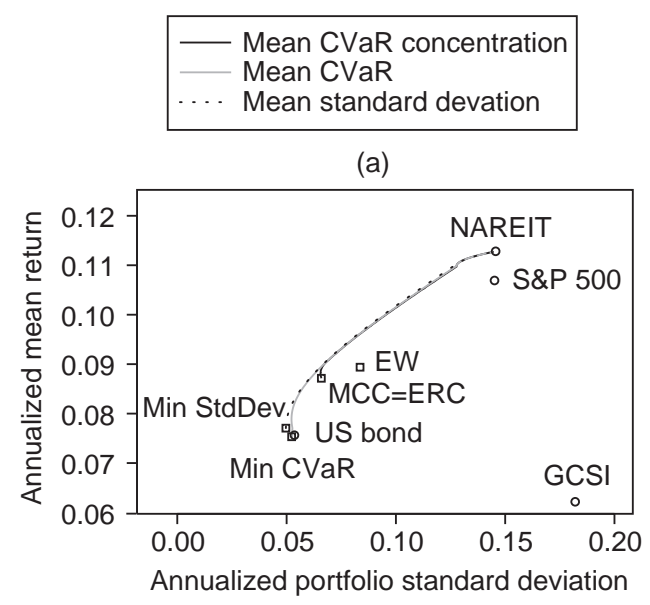

(b)

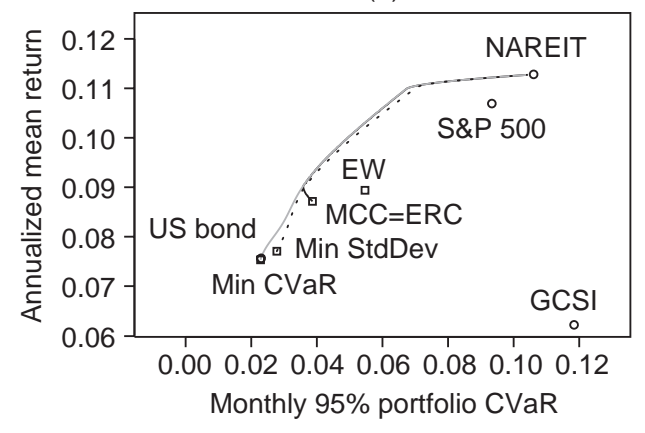

(c)

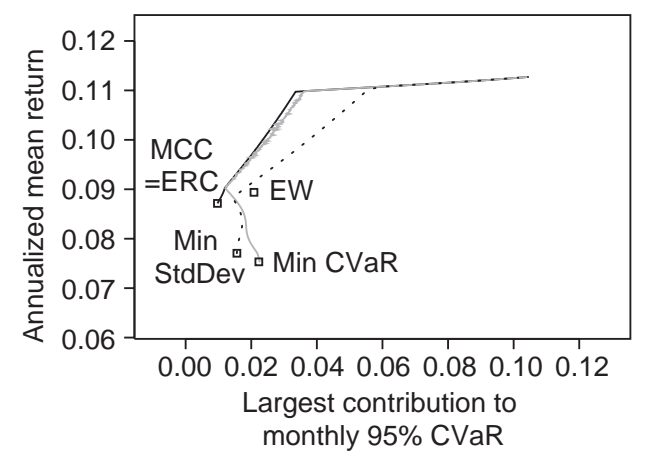




\subsection{Dynamic investment strategies}

Under a static risk budget allocation approach, the portfolio manager has determined a desired asset allocation and does not intend to stray far from it. However, it is wellknown that the risk and dependence of most financial assets are time-varying, which means that the optimal risk budget allocation portfolios also change over time.

Let us therefore consider a dynamic portfolio invested in bonds, equity, real estate and commodities. The portfolio is rebalanced quarterly to satisfy an equalweight, minimum CVaR or MCC objective. The CVaR budgets are computed by the Cornish-Fisher method in Boudt et al (2008), requiring an estimate of the first four (co)moments of the multivariate return distribution. Estimation of these moments is conditional on the information available at the time of rebalancing, as detailed in Appendix A.

Since we required a minimum sample size of eight years and the data span is January 1976-June 2010, the optimized weights are available for the quarters 1984 Q12010 Q3. First, we discuss the results for the equal-weight, minimum CVaR and MCC portfolios. Second, we analyze the sensitivity of the minimum CVaR and MCC portfolios to the inclusion of a weight or risk allocation constraint and the choice of risk measure. ${ }^{1}$

\subsubsection{Results for unconstrained portfolios}

Figure 4 on the facing page plots the weight and $\mathrm{CVaR}$ allocations of the equal-weight, minimum CVaR, and MCC portfolios. We find that, for almost all periods, the minimum CVaR portfolio is highly invested in the bond index, while the MCC portfolio is more balanced across all asset classes. As predicted by the theory, the risk allocation of the minimum CVaR portfolio coincides with its weight allocation. The risk allocation of the MCC portfolio is almost identical to the equal risk contribution state. The CVaR of the equal-weight portfolio is dominated by the S\&P 500, GSCI and NAREIT indexes, while the risk contribution of the bond is almost zero for many quarters. The reason for the bad performance of weight constraints in ensuring ex ante risk diversification is the nonlinear dependence of portfolio CVaR contributions on the weights. Reaching the portfolio manager's goal of ensuring risk diversification is therefore more efficiently achieved via direct constraints on the risk budget contributions rather than the weights.

\footnotetext{
${ }^{1}$ As noted by a referee, even though we evaluate the portfolios based on their performance, none of the objectives considered include performance (absolute or risk-adjusted). This is because of the difficulty in forecasting properly expected return, compared with forecasting risk, as already mentioned by Merton (1980).
} 
FIGURE 4 Stacked bar weight and CVaR contribution plots for the quarterly rebalanced equal-weight, minimum $\mathrm{CVaR}$ and minimum $\mathrm{CVaR}$ concentration portfolios.

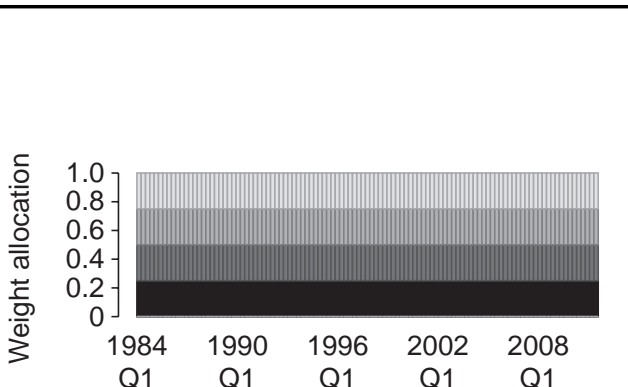

(a)

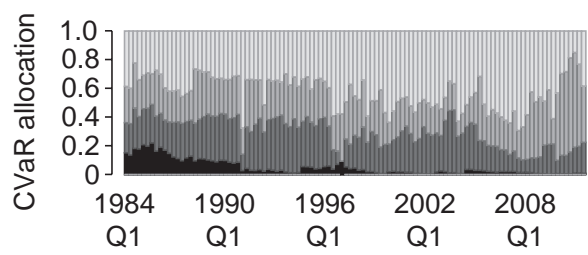

(b)
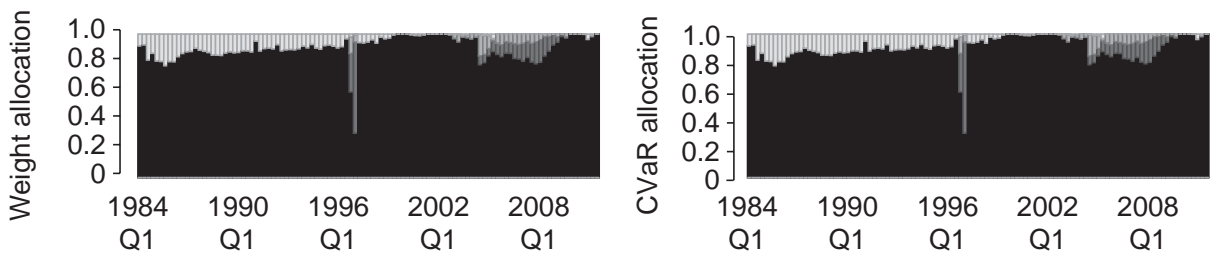

(c)
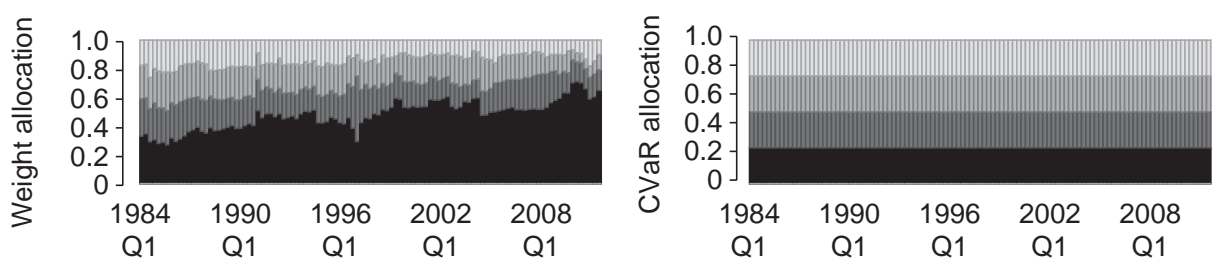

$$
\text { - US bond - S\&P } 500 \quad-\text { NAREIT - GSCI }
$$

(a) Equal weight. (b) Minimum CVaR. (c) Minimum CVaR concentration.

Part (a) in Figure 5 on the next page plots the ex ante portfolio risk estimates. As expected, the CVaR of the MCC portfolio is for all quarters in between the CVaR of the minimum CVaR portfolio and the CVaR of the equal-weight portfolio.

The solid gray and black lines in part (b) of Figure 5 on the next page plot the ratio of the monthly cumulative out-of-sample returns of the minimum CVaR and MCC portfolios versus the cumulative returns of the equal-weight portfolio over the period January 1984-June 2010. The value of the chart is less important than the slope of the 
FIGURE 5 Monthly CVaR of (a) the quarterly rebalanced equal-weight, minimum CVaR and minimum CVaR concentration portfolios and (b) the relative performance of the quarterly rebalanced minimum $\mathrm{CVaR}$ and minimum $\mathrm{CVaR}$ concentration portfolios versus the equal-weight portfolio.

(a)

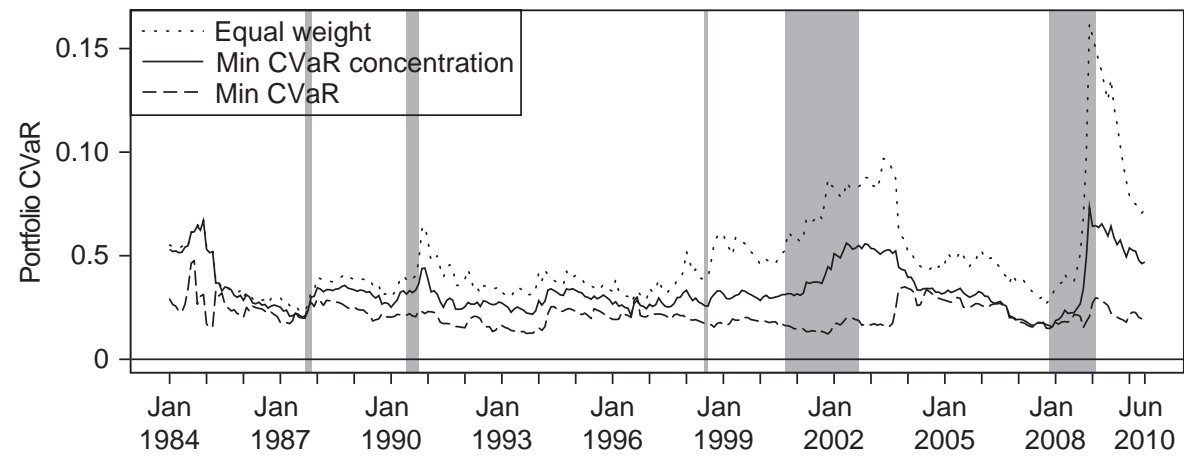

(b)

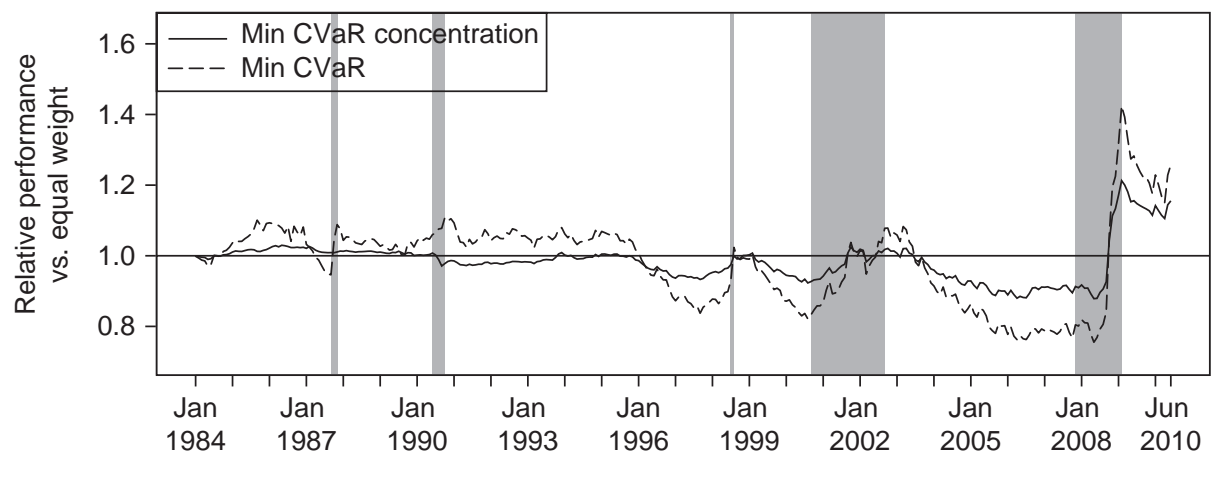

The shaded regions indicate a bear market regime.

line. If the slope is positive, the strategy in the numerator is outperforming the equalweight strategy, and vice versa. The vertical gray bars denote bear markets, defined by Ellis (2005) as periods with a decline in the S\&P 500 index of $12 \%$ or more. The left-hand side of the bar corresponds to the market peaks and the right-hand side to the stock market trough. We see in Figure 5 that the minimum CVaR portfolio, having a large allocation to bonds, outperforms the equal-weight and MCC portfolios at times of serious stock market downturn. The performance of the MCC portfolio seems to be a middle ground between the performance of the equal-weight and minimum $\mathrm{CVaR}$ portfolios. It offers an attractive compromise between the good performance of the 
minimum CVaR portfolio in adverse markets and the upward potential of the equalweight portfolio. A final observation is that periods where one strategy outperforms the other are relatively long and indicate the possibility of applying market timing strategies on top of these allocations.

Table 2 on the next page reports the annualized out-of-sample average return on the portfolios. When computed over the whole period, the minimum CVaR and MCC portfolios performed within 15 bps of one another. This is a small margin, given the long period of time presented. The risk statistics computed from the out-of-sample returns confirm the ex ante risk estimates from Figure 5 on the facing page. The value of the annualized standard deviation and monthly historical CVaR of the MCC portfolio is in between those of the minimum CVaR portfolio and the equal-weight portfolio. In the credit crisis the equal-weight portfolio suffered a drawdown of 52\%, which is significantly higher than the $34 \%$ drawdown of the MCC portfolio.

Splitting the sample into bull/bear periods, we see a much bigger variation in relative performance. The (annualized) return for the minimum CVaR portfolio trailed the MCC portfolio by more than 300 bps during equity bull markets, yet outperformed during bear markets by more than 1800 bps. The minimum CVaR and MCC portfolio therefore both have their appeal, depending on the market environment. This might lead to risk timing the portfolio allocation, whereby the investor selects their risk appetite based on broad market conditions. In a secular bull market, the investor might choose the MCC portfolio because of its relative outperformance in exchange for the risk of slightly larger losses. In a secular bear market, the minimum CVaR portfolio might be more appealing because of its conservatism.

Recall from (3.6) that the MCC portfolio is designed to have both a low downside risk and high downside risk diversification. To verify that the MCC effectively retains this property out-of-sample, we report in Table 2 on the next page the average Gini coefficient of the portfolio weights and the historical CVaR percentage concentration. The Gini coefficient takes values between 0 (equal-weight portfolio) and 1 (portfolio concentrated on one asset). ${ }^{2}$ We see that the minimum CVaR portfolio is heavily concentrated on a few assets compared with the MCC portfolio. The MCC portfolio thus strikes an attractive balance between a high risk adjusted return performance and a well-diversified portfolio. The historical CVaR percentage concentration is computed as the average proportion between the largest component loss contribution and the total loss for all losses that exceed the $5 \%$ quantile loss, computed using the returns from inception. We see that the equal-weight portfolio has the lowest $C V a R$ percentage concentration, but the highest CVaR. In contrast, the minimum CVaR portfolio has

${ }^{2}$ The Gini index is a measure of dispersion using the Lorenz curve. Let $z$ be a random variable on $[0,1]$ with distribution function $F$. The Gini index is calculated as $1-2 \int_{0}^{1} L(z) \mathrm{d} z$, where $L(z)=\int_{0}^{z} u \mathrm{~d} F(u) / \int_{0}^{1} u \mathrm{~d} F(u)$. 
TABLE 2 Summary statistics of monthly out-of-sample returns on equal-weight, minimum portfolio CVaR (concentration) investment strategies over the period January 1984-June 2010. [Table continues on next page.]

\begin{tabular}{|c|c|c|c|c|c|c|c|c|}
\hline \multirow{3}{*}{$\frac{\vec{D}}{\frac{D}{n}}$} & \multirow[b]{3}{*}{ Constraint } & \multicolumn{7}{|c|}{ Objective } \\
\hline & & \multirow[b]{2}{*}{$\begin{array}{l}\text { Equal } \\
\text { weight }\end{array}$} & \multirow[b]{2}{*}{$\begin{array}{c}\text { Min } \\
\text { CVaR }\end{array}$} & \multirow[b]{2}{*}{$\begin{array}{c}\text { Min CVaR } \\
\text { conc. }\end{array}$} & \multicolumn{3}{|c|}{ Min CVaR } & \multirow{2}{*}{$\begin{array}{l}\text { Min CVaR } \\
\text { conc. } \\
40 \% \text { position } \\
\text { limit }\end{array}$} \\
\hline & & & & & $\begin{array}{c}40 \% \\
\text { position } \\
\text { limit }\end{array}$ & $\begin{array}{c}40 \% \text { CVaR } \\
\text { allocation } \\
\text { limit }\end{array}$ & ERC & \\
\hline & \multicolumn{8}{|c|}{ (a) Full period (\%) } \\
\hline & Annual mean & 7.11 & 7.55 & 7.40 & 7.40 & 7.44 & 7.40 & 7.33 \\
\hline & Annual SD & 10.06 & 4.72 & 7.42 & 8.52 & 6.61 & 7.42 & 8.42 \\
\hline & Skewness & -3.14 & -0.49 & -2.35 & -2.36 & -1.92 & -2.35 & -2.69 \\
\hline & Excess kurtosis & 23.42 & 3.62 & 15.11 & 14.59 & 11.12 & 15.10 & 18.37 \\
\hline & Monthly historical CVaR & 7.55 & 2.62 & 5.14 & 6.17 & 4.51 & 5.14 & 6.08 \\
\hline & Gini portfolio weights & 0.00 & 0.68 & 0.25 & 0.23 & 0.47 & 0.25 & 0.17 \\
\hline & $\begin{array}{l}\text { Monthly historical CVaR } \\
\% \text { concentration }\end{array}$ & 0.63 & 0.96 & 0.62 & 0.71 & 0.76 & 0.62 & 0.61 \\
\hline$\stackrel{\underline{\varrho}}{c}$ & Portfolio turnover & 5.01 & 10.31 & 9.04 & 11.30 & 12.45 & 9.04 & 8.07 \\
\hline$\vec{v}$ & \multicolumn{8}{|c|}{ (b) Normal/bull stock market (\%) } \\
\hline$\frac{5}{3}$ & Annual mean & 12.64 & 8.02 & 11.33 & 12.19 & 10.42 & 11.33 & 11.96 \\
\hline$\stackrel{8}{\mathbb{D}}$ & Annual SD & 7.06 & 4.40 & 5.71 & 6.40 & 5.28 & 5.71 & 6.20 \\
\hline$\omega$ & Skewness & -0.30 & 0.12 & -0.13 & -0.26 & -0.08 & -0.13 & -0.23 \\
\hline 을. & Excess kurtosis & 0.84 & 1.57 & 0.39 & 1.10 & 1.18 & 0.39 & 0.74 \\
\hline$\overline{6}$ & Monthly historical CVaR & 3.51 & 2.07 & 2.65 & 3.06 & 2.42 & 2.65 & 3.00 \\
\hline
\end{tabular}


TABLE 2 Continued.

\begin{tabular}{|c|c|c|c|c|c|c|c|}
\hline \multirow[b]{3}{*}{ Constraint } & \multicolumn{7}{|c|}{ Objective } \\
\hline & \multirow[b]{2}{*}{$\begin{array}{c}\text { Equal } \\
\text { weight }\end{array}$} & \multirow[b]{2}{*}{$\begin{array}{c}\text { Min } \\
\text { CVaR }\end{array}$} & \multirow[b]{2}{*}{$\begin{array}{l}\text { Min CVaR } \\
\text { conc. }\end{array}$} & \multicolumn{3}{|c|}{ Min CVaR } & \multirow{2}{*}{$\begin{array}{c}\text { Min CVaR } \\
\text { conc. } \\
\text { 40\% position } \\
\text { limit }\end{array}$} \\
\hline & & & & $\begin{array}{l}40 \% \\
\text { position } \\
\text { limit }\end{array}$ & $\begin{array}{c}40 \% \text { CVaR } \\
\text { allocation } \\
\text { limit }\end{array}$ & ERC & \\
\hline \multicolumn{8}{|c|}{ (c) Bear stock market (\%) } \\
\hline Annual mean & -21.83 & 5.10 & -13.18 & -17.64 & -8.14 & -13.19 & -16.93 \\
\hline Annual SD & 17.10 & 6.14 & 11.52 & 13.37 & 10.18 & 11.53 & 13.60 \\
\hline Skewness & -2.59 & -1.46 & -2.56 & -2.30 & -2.18 & -2.56 & -2.54 \\
\hline Excess kurtosis & 9.65 & 4.33 & 8.40 & 7.18 & 5.93 & 8.40 & 8.82 \\
\hline Monthly historical CVaR & 15.44 & 4.31 & 11.06 & 12.63 & 9.57 & 11.06 & 12.80 \\
\hline \multicolumn{8}{|c|}{ (d) Drawdowns higher than $10 \%$} \\
\hline Credit crisis $^{1}$ & 0.52 & 0.10 & 0.34 & 0.41 & 0.27 & 0.34 & 0.42 \\
\hline $\begin{array}{l}\text { Asian-Russian } \\
\text { crisis }^{2}\end{array}$ & 0.15 & & & 0.11 & 0.10 & & 0.11 \\
\hline Black Monday $^{3}$ & 0.11 & & 0.11 & 0.11 & & 0.11 & 0.11 \\
\hline
\end{tabular}


the lowest $\mathrm{CVaR}$, but the highest $\mathrm{CVaR}$ percentage concentration. The MCC portfolio combines both a low CVaR concentration and low total portfolio CVaR.

Finally, we consider the portfolio turnover of the strategies, defined by DeMiguel et al (2009) as the average sum of the absolute value of the trades across the $N$ available assets:

$$
\text { turnover }=\frac{1}{T_{*}-1} \sum_{t=1}^{T_{*}-1} \sum_{i=1}^{N}\left|w_{(i) t+1}-w_{(i) t+}\right|,
$$

where $w_{(i) t+1}$ is the weight of asset $i$ at the start of rebalancing period $t+1, w_{(i) t+}$ is the weight of that asset before rebalancing at $t+1$ and $T_{*}$ is the total number of rebalancing periods. This turnover quantity can be interpreted as the average percentage of wealth traded in each period. The portfolio turnover is the lowest for the equal-weight portfolio (5.01\%). The MCC portfolio has a significantly lower turnover (9.04\%) than the minimum CVaR portfolio (10.31\%).

In conclusion, over the period January 1984-June 2010, the minimum CVaR portfolio has the lowest out-of-sample risk but a high risk concentration and turnover. The equal-weight strategy has the lowest turnover and risk concentration, but highest total risk. The proposed MCC portfolio is the second best in all these aspects. It achieves an attractive compromise between low overall risk, good upside return, high diversification and low turnover. When we condition on the market regime, we find that in bear markets, as expected, the minimum $\mathrm{CVaR}$ portfolio outperforms all strategies, but has a lower return in normal/bull markets than the equal-weight and MCC portfolios. Compared with the equal-weight diversification strategy, the MCC portfolio has a comparable return but lower risk in a normal/bull stock market, and its performance is less affected when the market regime switches to a more negative outlook. For this reason, we recommend a minimum CVaR allocation strategy in a bear market regime and the MCC strategy in a normal/bull market regime.

\subsubsection{Sensitivity to weight and CVaR allocation constraints}

The MCC portfolio directly optimizes the risk diversification of the portfolio. Previous research by Maillard et al (2010), Qian (2005) and Zhu et al (2010), however, has focused on using risk budgets as a constraint in the portfolio allocation, or imposing diversification through weight constraints. In Table 3 on the facing page, Figure 6 on page 62 and Figure 7 on page 63, we investigate the sensitivity of the portfolios to an equal risk contribution constraint, an upper $40 \%$ position limit or an upper $40 \%$ CVaR allocation limit. The choice of $40 \%$ is arbitrary, but it is consistent with the $40 \%$ allocation to equity in the stylized $60 / 40$ bond-equity portfolio.

Part (a) of Figure 6 on page 62 presents the weight allocations of the constrained minimum CVaR portfolios. We see that the $40 \%$ upper bound on the portfolio weights 
TABLE 3 Summary statistics of monthly out-of-sample returns on minimum portfolio standard deviation (concentration) investment strategies over the period January 1984June 2010.

\begin{tabular}{|c|c|c|c|c|c|c|}
\hline \multirow[b]{3}{*}{ Constraint } & \multicolumn{6}{|c|}{ Objective } \\
\hline & \multirow[b]{2}{*}{$\begin{array}{l}\text { Min } \\
\text { SD }\end{array}$} & \multirow{2}{*}{$\begin{array}{l}\text { Min SD } \\
\text { conc. } \\
40 \% \text { pos }\end{array}$} & \multirow[b]{2}{*}{$\begin{array}{l}40 \% \text { SD } \\
\text { limit }\end{array}$} & \multicolumn{2}{|l|}{ Min SD } & \multirow{2}{*}{$\begin{array}{l}\text { Min SD } \\
\text { conc. } \\
\text { 40\% pos } \\
\text { limit }\end{array}$} \\
\hline & & & & $\begin{array}{c}\text { allocation } \\
\text { limit }\end{array}$ & ERC & \\
\hline \multicolumn{7}{|c|}{ (a) Full period (\%) } \\
\hline Annual mean & 7.48 & 7.30 & 6.96 & 7.21 & 7.30 & 7.17 \\
\hline Annual SD & 4.62 & 7.02 & 8.18 & 6.13 & 7.02 & 8.22 \\
\hline Skewness & -0.42 & -2.48 & -2.50 & -1.73 & -2.48 & -2.89 \\
\hline Excess kurtosis & 1.86 & 17.20 & 16.99 & 10.55 & 17.19 & 21.36 \\
\hline Monthly historical CVaR & 2.37 & 4.82 & 5.80 & 3.85 & 4.82 & 5.93 \\
\hline $\begin{array}{l}\text { Gini portfolio } \\
\text { weights }\end{array}$ & 0.62 & 0.28 & 0.28 & 0.46 & 0.28 & 0.18 \\
\hline $\begin{array}{l}\text { Monthly historical CVaR } \\
\% \text { conc }\end{array}$ & 0.90 & 0.64 & 0.65 & 0.76 & 0.64 & 0.61 \\
\hline Portfolio turnover & 11.79 & 8.63 & 14.90 & 12.97 & 8.64 & 7.61 \\
\hline
\end{tabular}

(b) Normal/bull stock market (\%)

\begin{tabular}{lrrrrrr}
\hline Annual mean & 7.99 & 10.66 & 10.57 & 9.10 & 10.67 & 11.35 \\
Annual SD & 4.39 & 5.45 & 6.41 & 5.27 & 5.46 & 6.08 \\
Skewness & -0.05 & -0.20 & -0.39 & -0.27 & -0.20 & -0.27 \\
Excess kurtosis & 0.34 & 0.22 & 0.98 & 0.07 & 0.22 & 0.66 \\
Monthly historical CVaR & 2.02 & 2.52 & 3.17 & 2.61 & 2.52 & 2.93 \\
\hline
\end{tabular}

(c) Bear stock market (\%)

\begin{tabular}{lrrrrrr}
\hline Annual mean & 4.83 & -10.32 & -11.94 & -2.71 & -10.33 & -14.71 \\
Annual SD & 5.63 & 11.07 & 13.01 & 8.96 & 11.07 & 13.56 \\
Skewness & -1.16 & -2.70 & -2.63 & -2.52 & -2.70 & -2.71 \\
Excess kurtosis & 3.46 & 10.11 & 9.78 & 9.78 & 10.10 & 10.40 \\
Monthly historical CVaR & 3.51 & 9.97 & 12.08 & 7.64 & 9.97 & 12.53 \\
\hline
\end{tabular}

(d) Drawdowns higher than $10 \%$

\begin{tabular}{llllll}
\hline Credit crisis $^{1}$ & 0.33 & 0.41 & 0.25 & 0.33 & 0.42 \\
Asian-Russian $_{\text {crisis }^{2}}$ & 0.11 & 0.17 & 0.14 & 0.11 & 0.12 \\
\hline
\end{tabular}

1 June 2008-February 2009. ${ }^{2}$ November 1997-August 1998 for the minimum standard deviation concentration and ERC constrained portfolios. Otherwise November 1997-February 1998. 
FIGURE 6 Stacked bar weight and CVaR contribution plots for the constrained minimum $\mathrm{CVaR}$ and minimum CVaR concentration portfolios invested in the Merrill Lynch US bond, S\&P 500, NAREIT and S\&P GSCI indexes.

(a)
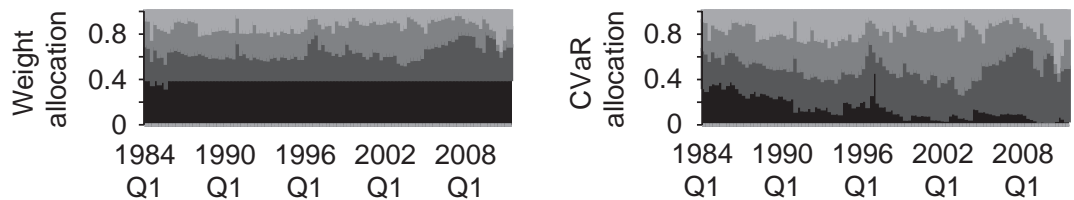

(b)
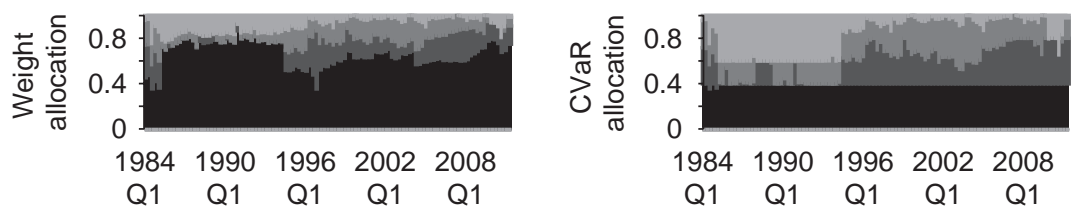

(c)
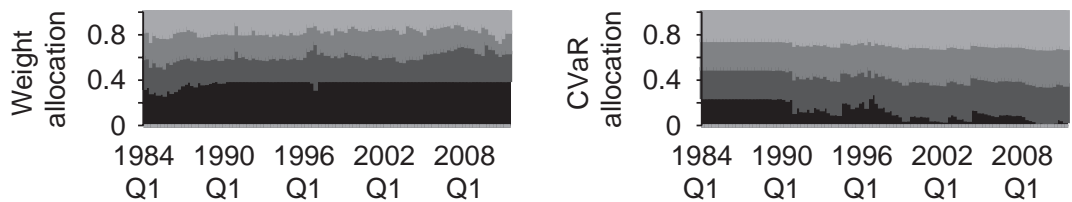

$$
\text { - US bond - S\&P } 500 \quad-\text { NAREIT }- \text { GSCI }
$$

The portfolios are rebalanced quarterly. (a) Min CVaR $+40 \%$ position limit. (b) Min CVaR $+40 \%$ CVaR allocation limit. (c) Min CVaR concentration $+40 \%$ position limit.

and risk allocations is stringent for almost all periods. Under these constraints, the component $\mathrm{CVaR}$ contribution of the minimum CVaR portfolio no longer coincides with the weight allocation. The investment in the bond typically contributes less to $\mathrm{CVaR}$ risk than its portfolio weight. Its contribution is for some months even negative under the position limit. Part (c) of Figure 6 shows the weight and risk allocation of the MCC portfolio under a $40 \%$ upper bound on the portfolio weights. We see that, in spite of the weight constraint, the risk of the MCC portfolio is still more equally spread out than for the minimum CVaR portfolio, where for some periods the S\&P 500 investment causes more than half of portfolio risk. 
FIGURE 7 Monthly CVaR of (a) the quarterly rebalanced constrained minimum CVaR and minimum CVaR concentration portfolios and (b) the relative performance of the quarterly rebalanced constrained minimum $\mathrm{CVaR}$ and minimum $\mathrm{CVaR}$ concentration portfolios versus the equal-weight portfolio.

(a)

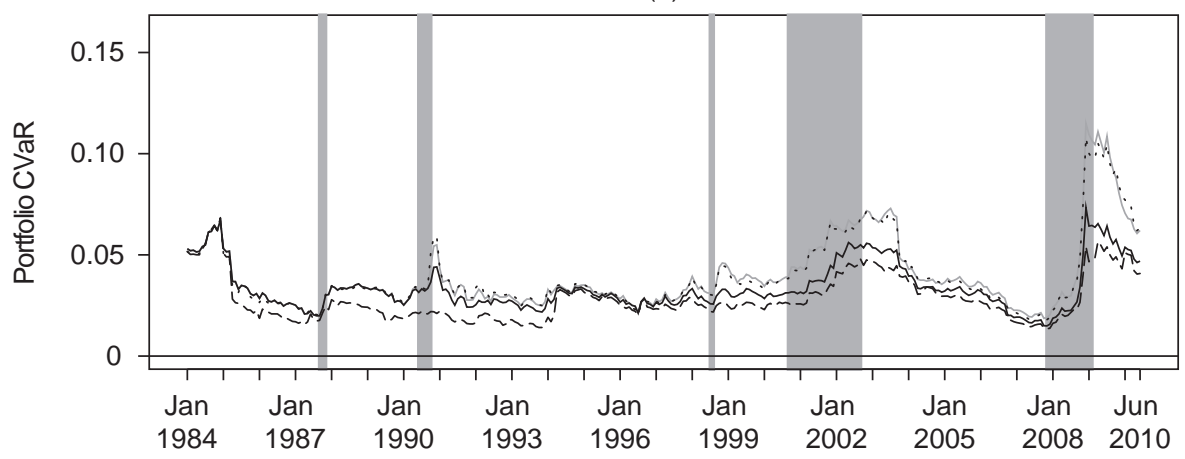

(b)

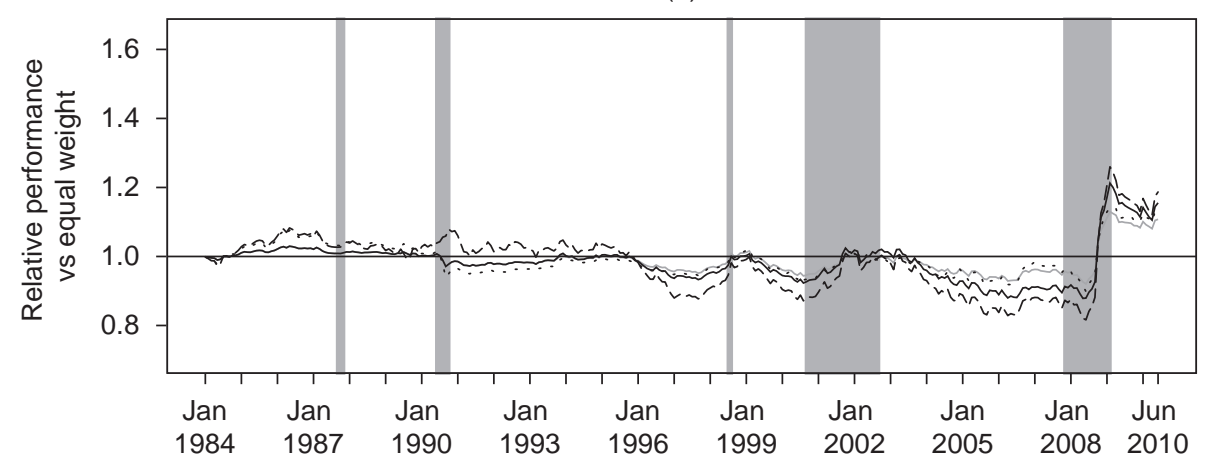

The shaded regions indicate a bear market regime.

From the weight and CVaR allocation plots, it is clear that adding position or risk allocation limits pushes the minimum CVaR and MCC portfolio toward an allocation that is closer to the equal-weight portfolio. Consequently, the return, risk and turnover properties of these constrained portfolios are closer to the equal-weight portfolio, as can be seen in Table 3 on page 61 and Figure 7. Compared with the $40 \%$ CVaR alloca- 
tion limit, the maximum $40 \%$ weight constraint leads to portfolios with a substantially higher predicted risk (see part (a) of Figure 6 on page 62) and realized risk (see the standard deviation and historical CVaR values in Table 3 on page 61). In all aspects, the unconstrained MCC portfolio and ERC constrained minimum CVaR portfolios are very similar.

\subsubsection{Sensitivity to the choice of risk measure}

In Maillard et al (2010), Qian (2005) and Zhu et al (2010), the portfolio standard deviation is used as the risk measure in the risk allocation portfolio. We analyze the sensitivity of our results to the use of downside risk measures in Table 3 on page 61, where we report the performance statistics for the same investment styles, but using the portfolio standard deviation as the risk measure. Overall, it seems that, for this investment universe, changing the risk measure only has a marginal impact on the out-of-sample performance, compared with the choice of investment style. In fact, when testing for the equality of the Sharpe ratios between the minimum CVaR (concentration) and minimum standard deviation (concentration) portfolios using the test of Jobson and Korkie (1981) and Memmel (2003), we do not find a significant difference in performance at a $90 \%$ confidence level. Similarly to the CVaR risk measure, we find that the minimum standard deviation concentration portfolio constitutes an attractive middle ground between the upward potential of the equalweight portfolio in normal/bull markets and the low risk of the minimum standard deviation portfolio.

\section{CONCLUSION}

An extensive empirical application of ex ante downside risk budget methods to dynamic allocation across bonds, commodities, domestic and international equity illustrated the out-of-sample effectiveness of downside risk budgets in generating portfolios that have low portfolio risk and risk concentration, high diversification and low portfolio turnover. A first strategy is to impose bound constraints on the percentage CVaR contributions. This provides a direct substitute and improvement to the commonly practiced risk diversification approach based on position limits. A second strategy consists of minimizing the largest component CVaR contribution, which directly addresses risk diversification, even in portfolios with nonnormally distributed assets. The properties of these approaches as described in this paper compare favorably to the equal-weight portfolio. Unconstrained, the minimum CVaR concentration portfolio is typically similar to the equal risk contribution portfolio of Qian (2005). Furthermore, it may be easily combined with many other investor objectives and constraints (such as return targets or drawdown and cardinality constraints). Investors can thus optimally balance their maximum return, minimum downside risk and maximum 
downside risk diversification objectives through an ex ante use of CVaR budgets in portfolio optimization. Based on our empirical study, we recommend the optimization of the asset allocation according to a minimum CVaR objective in a bear market regime and the minimization of the portfolio's CVaR concentration in a normal/bull market regime.

There are several interesting directions for further research. First of all, the risk allocation methodology could be applied to a large-scale equity portfolio, in which case we recommend the formulation of the risk budget constraints and objectives at a more aggregate level, such as at the level of the country and industry rather than the individual stock. A second topic for further research is the choice of downside risk estimator and the loss probability at which the downside risk is computed. Finally, a switching allocation rule between a low-risk strategy and a strategy that combines risk objectives with views on the returns also merits further research.

\section{APPENDIX A}

\section{A.1 CVaR budget of the fully invested minimum CVaR portfolio}

The Langrangian associated with the fully invested minimum CVaR portfolio is

$$
\operatorname{CVaR}_{w}(\alpha)+\lambda\left(w^{\prime} \iota-1\right)
$$

with $\iota$ the $N \times 1$ vector with each element equal to 1 . From the first order conditions, it follows that

$$
\left.\frac{\partial \operatorname{CVaR}_{w}(\alpha)}{\partial w}\right|_{w=w^{\min C V a R}}=-\lambda \iota .
$$

Hence, the partial derivatives should all be equal, and because

$$
C_{(i)} \operatorname{CVaR}_{w}(\alpha)=w_{(i)} \frac{\partial \operatorname{CVaR}_{w}(\alpha)}{\partial w_{(i)}},
$$

there exists a unique constant $k$ such that

$$
w_{(i)}^{\min C \operatorname{CVR}}=k C_{(i)} \operatorname{CVaR}_{w^{\min \operatorname{CVaR}}}(\alpha),
$$

for all $i=1, \ldots, N$. Because of the full investment constraint, the weights need to sum up to unity, which is satisfied for $k=1 / \mathrm{CVaR}_{w^{\operatorname{minCVaR}}}(\alpha)$. Replacing $k$ with $1 / \mathrm{CVaR}_{w^{\operatorname{minCVa}}}(\alpha)$ in (A.3) yields that the weight and percentage CVaR allocation coincide for the minimum CVaR portfolio.

\section{A.2 Implementation of the CVaR estimator}

The CVaR estimator requires an estimate of the $N \times 1$ conditional mean $\mu_{t}$, the $N \times N$ conditional covariance matrix $\Sigma_{t}$, the $N \times N^{2}$ conditional coskewness $\Xi_{t}$, and the 
$N \times N^{3}$ cokurtosis matrix $\Psi_{t}$. We obtain estimates of these conditional (co)moments through the following stepwise estimation procedure.

(1) Estimate the conditional mean monthly return $\mu_{t}$ as the sample average of the returns from inception up to month $t-1$.

(2) Center the monthly returns in the sample around $\mu_{t}: \bar{r}_{s}=r_{s}-\mu_{t}$ for all $s=1, \ldots, t-1$.

(3) Estimate the conditional covariance matrix for each return in the sample, as well as the conditional covariance matrix for next month's return $\left(\Sigma_{t}\right)$, using the DCC-GARCH(1,1) model of Engle (2002). Under this approach, the parameters of the individual GARCH $(1,1)$ volatilities and conditional correlations are estimated separately, using a two-stage quasi-maximum likelihood estimation procedure with variance and correlation targeting. The conditional covariances are then computed as products of individual volatilities and conditional correlations.

(4) For the returns in the sample, compute the devolatilized centered return series: $u_{s}=\sum_{s}^{-1 / 2} \bar{r}_{s}$ for all $s=1, \ldots, t-1$.

(5) Use the Winsorization procedure of Boudt et al (2008) to reduce the magnitude of the most extreme devolatilized return vectors in the sample.

(6) Compute the equicorrelation estimator of $\Xi_{t}$ and $\Psi_{t}$ on the sample of Winsorized devolatilized returns, as proposed by Martellini and Ziemann (2010).

Given a portfolio weight vector $w$, the second, third and fourth portfolio moments can then be computed as

$$
m_{2 t}=w^{\prime} \Sigma_{t} w, \quad m_{3 t}=w^{\prime} \Xi_{t}(w \otimes w), \quad m_{4 t}=w^{\prime} \Psi_{t}(w \otimes w \otimes w),
$$

respectively, where $\otimes$ denotes the Kronecker product. The portfolio skewness $s_{t}=$ $m_{3 t} / m_{2 t}^{3 / 2}$ and the excess kurtosis $k_{t}=m_{4 t} / m_{2 t}^{2}-3$.

The Cornish-Fisher-based CVaR estimator proposed by Boudt et al (2008) is derived from the Cornish-Fisher expansion. For a zero mean, unit variance random variable with skewness $s_{t}$ and excess kurtosis $k_{t}$, the second order Cornish-Fisher expansion of the quantile function around the Gaussian quantile function $\Phi^{-1}(\cdot)$ equals

$$
g_{\alpha t}=z_{\alpha}+\frac{1}{6}\left(z_{\alpha}^{2}-1\right) s_{t}+\frac{1}{24}\left(z_{\alpha}^{3}-3 z_{\alpha}\right) k_{t}-\frac{1}{36}\left(2 z_{\alpha}^{3}-5 z_{\alpha}\right) s_{t}^{2},
$$

with $z_{\alpha}=\Phi^{-1}(\alpha)$. The Cornish-Fisher CVaR for the portfolio return in month $t$ is then given by

$$
\operatorname{CVaR}_{t}(\alpha)=-w^{\prime} \mu_{t}+\sqrt{m_{2 t}} d_{t}(\alpha)
$$


with

$$
\begin{aligned}
d_{t}(\alpha)=\frac{1}{\alpha}\left\{\phi\left(g_{\alpha t}\right)+\frac{1}{24}\left[I^{4}-6 I^{2}+\right.\right. & \left.3 \phi\left(g_{\alpha t}\right)\right] k_{t}+\frac{1}{6}\left[I^{3}-3 I^{1}\right] s_{t} \\
& \left.+\frac{1}{72}\left[I^{6}-15 I^{4}+45 I^{2}-15 \phi\left(g_{\alpha t}\right)\right] s_{p}^{2}\right\}
\end{aligned}
$$

and where

$$
I^{q}= \begin{cases}\sum_{i=1}^{q / 2}\left(\frac{\prod_{j=1}^{q / 2} 2 j}{\prod_{j=1}^{i} 2 j}\right) g_{\alpha t}^{2 i} \phi\left(g_{\alpha t}\right)+\left(\prod_{j=1}^{q / 2} 2 j\right) \phi\left(g_{\alpha t}\right) & \text { for } q \text { even, } \\ \sum_{i=0}^{q^{*}}\left(\frac{\prod_{j=0}^{q^{*}}(2 j+1)}{\prod_{j=0}^{i}(2 j+1)}\right) g_{\alpha t}^{2 i+1} \phi\left(g_{\alpha t}\right)-\left(\prod_{j=0}^{q^{*}}(2 j+1)\right) \Phi\left(g_{\alpha t}\right) & \text { for } q \text { odd },\end{cases}
$$

$q^{*}=(q-1) / 2$ and $\phi(\cdot)$ and $\Phi(\cdot)$ are the standard normal density and cumulative distribution functions, respectively.

Although the CVaR estimation formulas seem rather complex, they lend themselves to efficient translation into a simple algorithm that computes both CVaR and component CVaR in less than a second, even for portfolios with a large number of assets. $^{3}$

\section{REFERENCES}

Ardia, D., Boudt, K., Carl, P., Mullen, K., and Peterson, B. (2011). Differential evolution (DEoptim) for non-convex portfolio optimization. $R$ Journal 3(1), 27-34.

Artzner, P., Delbaen, F., Eber, J. M., and Heath, D. (1999). Coherent measures of risk. Mathematical Finance 9(3), 203-228.

Boudt, K., Peterson, B. G., and Croux, C. (2008). Estimation and decomposition of downside risk for portfolios with non-normal returns. The Journal of Risk 11(2), 79-103.

Boudt, K., Carl, P., and Peterson, B. G. (2011). PortfolioAnalytics: portfolio analysis, including numeric methods for optimization of portfolios. R Package, Version 0.6.1.

Carl, P., and Peterson, B. G. (2010). PerformanceAnalytics: econometric tools for performance and risk analysis. $R$ Package, Version 1.0.2.1.

Choueifaty, Y., and Coignard, Y. (2008). Toward maximum diversification. Journal of Portfolio Management 35(1), 10-24.

Chow, G., and Kritzman, M. (2001). Risk budgets. Journal of Portfolio Management 27(2), 56-60.

DeMiguel, V., Garlappi, L., and Uppal, R. (2009). Optimal versus naïve diversification: how inefficient is the $1 / N$ portfolio strategy? Review of Financial Studies 22(5), 1915-1953.

Denault, M. (2001). Coherent allocation of risk capital. The Journal of Risk 4(1), 1-33.

Ellis, J. (2005). Ahead of the Curve: A Commonsense Guide to Forecasting Business and Market Cycles. Harvard Business Press, Cambridge, MA.

\footnotetext{
${ }^{3}$ The estimator is available in Carl and Peterson's (2010) R package PerformanceAnalytics.
} 
Engle, R. (2002). Dynamic conditional correlation: a simple class of multivariate generalised autoregressive conditional heteroskedasticity models. Journal of Business and Economic Statistics 20(3), 339-350.

Jobson, J., and Korkie, B. (1981). Performance hypothesis testing with the Sharpe and Treynor measures. Journal of Finance 36(4), 889-908.

Keel, S., and Ardia, D. (2011). Generalized marginal risk. Journal of Asset Management 12(2), 123-131.

Lee, W. (2011). Risk-based asset allocation: a new answer to an old question? Journal of Portfolio Management 37(4), 11-28.

Litterman, R. B. (1996). Hot spots ${ }^{\mathrm{TM}}$ and hedges. Journal of Portfolio Management 22(5), $52-75$.

Maillard, S., Roncalli, T., and Teiletche, J. (2010). On the properties of equally-weighted risk contributions portfolios. Journal of Portfolio Management 36(4), 60-70.

Martellini, L., and Ziemann, V. (2010). Improved forecasts of higher-order comoments and implications for portfolio selection. Review of Financial Studies 23(4), 1467-1502.

Memmel, C. (2003). Performance hypothesis testing with the Sharpe ratio. Finance Letters 1(1), 21-23.

Merton, R. (1980). On estimating the expected return on the market. Journal of Financial Economics 8(4), 323-361.

Pearson, N. D. (2002). Risk Budgeting: Portfolio Problem Solving with Value-at-Risk, 1st edn. Wiley.

Peterson, B. G., and Boudt, K. (2008). Component VAR for a non-normal world. Risk 21(11), 78-81.

Pflug, G. C. (2000). Some remarks on the value-at-risk and the conditional value-at-risk. In Probabilistic Constrained Optimization: Methodology and Applications, Uryasev, S. (ed), pp. 272-281. Kluwer, Dordrecht.

Price, K., Storn, R., and Lampinen, J. (2005). Differential Evolution: A Practical Approach to Global Optimization. Springer.

Qian, E. (2005). Risk parity portfolios: efficient portfolios through true diversification of risk. Report, Panagora Asset Management (September).

Rockafellar, R. T., and Uryasev, S. (2000). Optimization of conditional value-at-risk. The Journal of Risk 2(3), 21-41.

Scaillet, O. (2002). Nonparametric estimation and sensitivity analysis of expected shortfall. Mathematical Finance 14(1), 74-86.

Scherer, B. (2007). Portfolio Construction and Risk Budgeting, 3rd edn. Risk Books, London.

Stoyanov, S., Rachev, S. T., and Fabozzi, F. J. (2009). Sensitivity of portfolio VaR and CVaR to portfolio return characteristics. Working Paper, Universität Karlsruhe.

Topaloglou, N., Vladimirou, H., and Zenios, S. A. (2002). CVaR models with selective hedging for international asset allocation. Journal of Banking and Finance 26(7), 1535-1561.

Zhu, S., Li, D., and Sun, X. (2010). Portfolio selection with marginal risk control. The Journal of Computational Finance 14(1), 3-28. 